\title{
Palladium Catalysis in the Synthesis of Hydroxymethylfuryl Pyrimidinyl Ketones
}

\author{
Joseph Arukwe and Kjell Undheim \\ Department of Chemistry, University of Oslo, N-0315 Oslo 3, Norway
}

\begin{abstract}
Arukwe, J. and Undheim, K., 1991. Palladium Catalysis in the Synthesis of Hydroxymethylfuryl Pyrimidinyl Ketones. - Acta Chem. Scand. 45: 914-918.

Furylmethyl silyl ethers have been stannylated by means of metal-metal exchange from the corresponding lithiofurans. Unsymmetrical heteroaryl ketones are formed in palladium-catalyzed coupling reactions between the stannylfurans and 2-methylthiopyrimidine-5-carbonyl chloride. Oxidation of the sulfenyl group and subsequent hydrolysis yield 5-furoyl-2(1H)-pyrimidinones.
\end{abstract}

5-Acylpyrimidines had received little attention up to the time when our study of methods for their preparation was started in order to make 5-acyl derivatives of pyrimidine systems available for biological evaluations. ${ }^{1-3}$ We are looking for compounds that will reversibly arrest the mitosis of cells in metaphase. Such compounds may be useful as growth-regulating adjuvants when used together with phase-specific antineoplastic drugs in treatment of diseases caused by uncontrolled, rapidly proliferating cells. ${ }^{4}$ Several $\mathrm{N}$-1 substituted 5-halogeno-2(1H)-pyrimidinones are able to cause reversible arrest of cells in metaphase ${ }^{4,5}$ Screening of 5-acylpyrimidinones has shown that certain 5-acyl derivatives of pyrimidinones have similar effects on the cell cycle. ${ }^{6}$ 5-Acyl derivatives are particularly attractive because of the possibility for fine manipulation of the electronwithdrawing effect from the acyl group by appropriate substitutions in the acyl moiety. In this report we describe methods for introduction of hydroxyalkylfuranoyl substituents into the pyrimidine 5-position using palladium-mediated coupling reactions between stannylfurans and a pyrimidine-5-carbonyl chloride.

Scheme 1 shows the preparation of 5-trimethylstannyl derivatives of silyl protected 2- and 3-furylmethanol from the corresponding lithiofurans by metal-metal exchange. Since the hydrolytic stability of silyl ethers increases with the bulkiness of the silyl substituents, ${ }^{7}$ dimethylthexylsilyl (DMTS) ethers were used. These were prepared from the silyl chloride and the hydroxymethylfuran in pyridine solution in the presence of $4-N, N$-dimethylaminopyridine (DMAP).

The silyl ether of 2-furylmethanol was lithiated in the 5 -position with butyllithium at $-60^{\circ} \mathrm{C}$. Lithiation of 3furylmethanol and silyl ethers is reported to yield a 2 - and 5 -lithiofuran isomer mixture. ${ }^{8,9}$ Selective lithiation of the<smiles>O=C([O-])c1coc(Br)c1</smiles>

$1 \mathbf{a}$

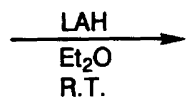

R.T.

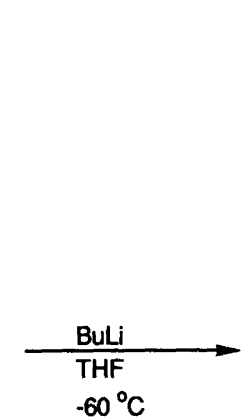

4b<smiles>COc1ccco1</smiles><smiles>OCc1coc(Br)c1</smiles>

2a
R.T.

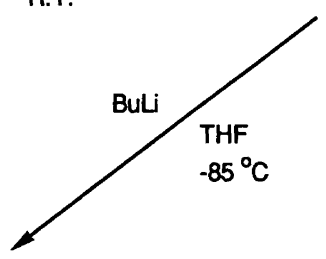<smiles>COCc1coc(Br)c1</smiles>

3a $-60^{\circ} \mathrm{C}$<smiles></smiles>

5

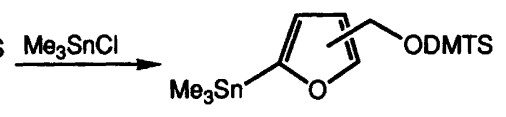

6 a 3,5 - isomer 


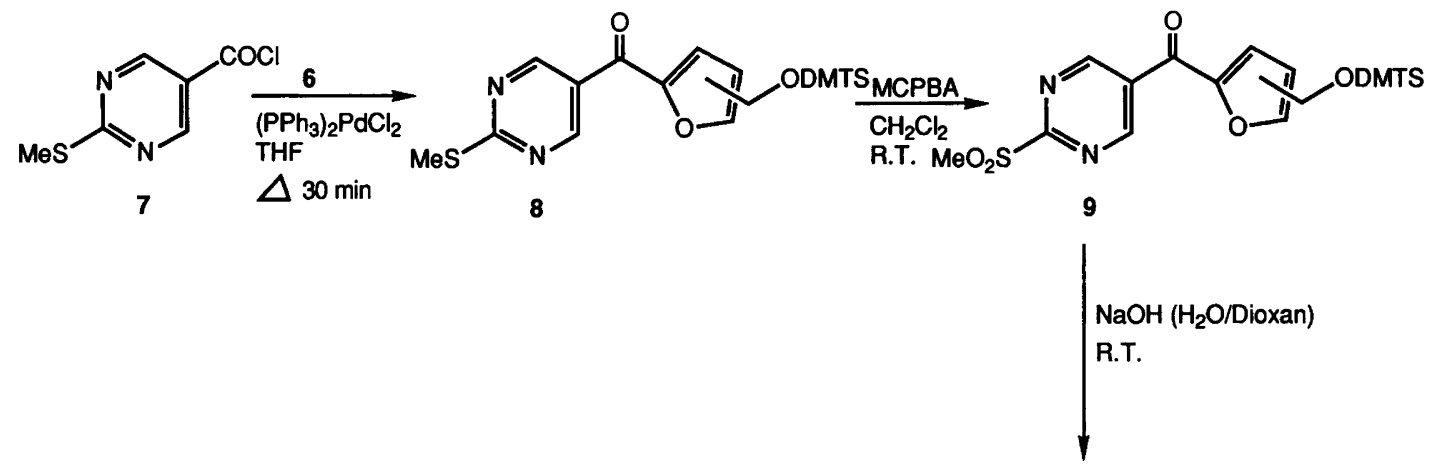

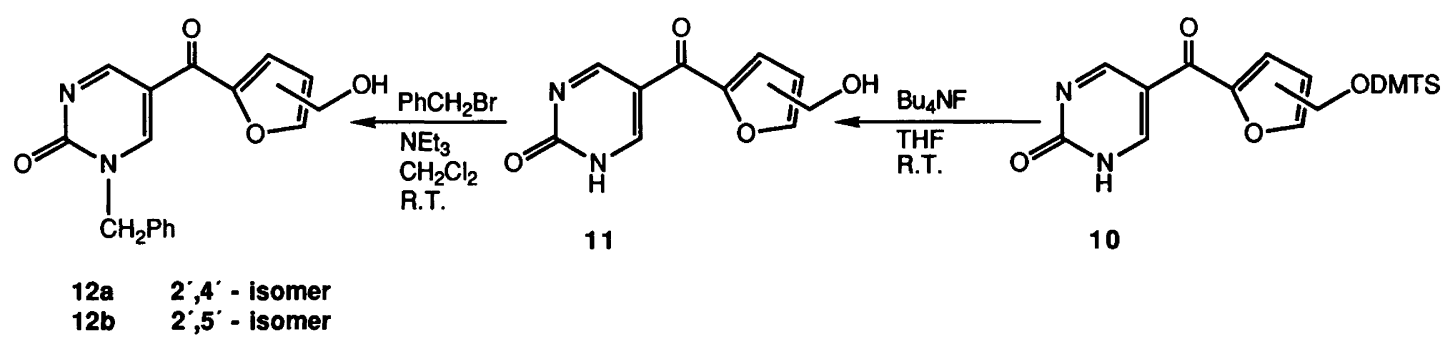

\section{Scheme 2 .}

5-position in the 3-furylmethanol series was achieved by a halogen-metal exchange reaction between the bromo derivative $3 \mathrm{a}$ and butyllithium at $-85^{\circ} \mathrm{C}$. The starting material in this series was 3-furylcarboxylic acid which was brominated in the 5-position. Literature methods failed to give both satisfactory yield and isomer purity of the desired product $1 \mathrm{a},{ }^{10}$ which was best obtained by the treatment of 3-furancarboxylic acid with $\mathrm{N}$-bromosuccinimide in acetic acid at ambient temperature. Reduction of the bromo acid 1a with lithium aluminum hydride (LAH) gave the 3-furylmethanol 2a. ${ }^{11}$ Quenching of the lithiofuran 5 with trimethylstannyl chloride gave the 5-stannylfuran derivative 6.

For the coupling reaction 2-methylthiopyrimidine-5-carboxylic acid was chosen because it is readily available from a cyclisation reaction from simple starting materials. ${ }^{2}$ The pyrimidine-5-carboxylic acid as its carbonyl chloride 7 in tetrahydrofuran (THF), was coupled wih the stannylfuran 6 using bis(triphenylphosphine)palladium(II) dichloride as a catalyst. The silyl ether function was not attacked by the stannyl chloride generated in the coupling reaction; for comparison, the tert-butyldimethylsilyl group on the pyrimidine-2-oxygen is cleaved under similar reaction conditions. ${ }^{3}$ For the chemoselective oxidation of the sulfide function to the sulfone $9 m$-chloroperbenzoic acid (MCPBA) was used. Hydrolytic removal of the sulfonyl group proceeded readily under mild alkaline conditions; the hydrolysis is facilitated by the 5-acyl group (para-position). The 3-furylmethanol 11 was generated from its silyl protected precursor 10 by treatment of the latter with tetrabutylammonium fluoride in THF under anhydrous conditions. Finally the 5-furylpyrimidinone 12 was prepared by $N$-alkylation of 11 as its triethylamine salt with benzyl bromide.

\section{Experimental}

The ${ }^{1} \mathrm{H}$ and the ${ }^{13} \mathrm{C}$ NMR spectra were recorded in $\mathrm{CDCl}_{3}$ at 60 and $75 \mathrm{MHz}$ respectively, unless otherwise specified. The mass spectra were recorded under electron impact conditions at $70 \mathrm{eV}$ ionization voltage. Isobutane or ammonia was used for chemical ionization $(\mathrm{Cl})$.

5-Bromo-3-furancarboxylic acid (1a). $\mathrm{N}$-Bromosuccinimide $(3.20 \mathrm{~g}, 18.0 \mathrm{mmol})$ was added with stirring to a solution of 3-furancarboxylic acid in acetic acid $(10 \mathrm{ml})$ at ambient temperature. The reaction was weakly exothermic. The mixture was stirred at ambient temperature for $24 \mathrm{~h}$ and evaporated. The residual oil was extracted with diethyl ether, leaving behind the succinimide, and the ether solution evaporated. The residual reddish oil was triturated with water and the resultant solid crystallized from water; yield $1.40 \mathrm{~g}(41 \%)$, m.p. $136^{\circ} \mathrm{C} .{ }^{9 \mathrm{a}}{ }^{1} \mathrm{H}$ NMR: $\delta 6.70(\mathrm{H}-4)$, $8.10(\mathrm{H}-2)$.

5-Bromo-3-furylmethanol (2a). A solution of 5-bromo-3furancarboxylic acid $(1.58 \mathrm{~g}, 8.27 \mathrm{mmol})$ in dry diethyl ether $(15 \mathrm{ml})$ was added dropwise with stirring to a solution of lithium aluminum hydride $(0.34 \mathrm{~g}, 9.0 \mathrm{mmol})$ in dry diethyl ether $(15 \mathrm{ml})$ at ambient temperature. The mixture was stirred for $15 \mathrm{~min}$, after which $2 \mathrm{M} \mathrm{NaOH}(7 \mathrm{ml})$ was added. The two phases were separated and the ether solution was washed, dried $\left(\mathrm{MgSO}_{4}\right)$ and evaporated to give the product; yield $0.68 \mathrm{~g}(74 \%)$. ${ }^{1} \mathrm{H}$ NMR: $\delta 2.70(\mathrm{OH})$, $4.50\left(\mathrm{CH}_{2}\right), 6.35(\mathrm{H}-4), 7.40(\mathrm{H}-2) .{ }^{10}$

5-Bromo-3-(dimethylthexylsilyloxymethyl)furan (3a). Dimethylthexylsilyl chloride $(1.38 \mathrm{~g}, 7.40 \mathrm{mmol})$ was added dropwise with stirring to a solution of 5-bromo-3-furyl- 
methanol $(1.31 \mathrm{~g}, 7.40 \mathrm{mmol})$, triethylamine $(0.91 \mathrm{~g}, 9.0$ mmol) and DMAP $(0.2 \mathrm{~g})$ in dichloromethane $(10 \mathrm{ml})$ at ambient temperature. The mixture was stirred at ambient temperature for $24 \mathrm{~h}$ when TLC monitoring showed the reaction to be complete. The mixture was filtered and the solid was washed with dichloromethane. The filtrate and washings were shaken with water $(3 \times 30 \mathrm{ml})$ and the organic solution was dried $\left(\mathrm{MgSO}_{4}\right)$ and evaporated. The residual oil was purified by flash chromatography on silica gel using light petroleum-EtOAc (7:3); the product was a colourless oil, $1.90 \mathrm{~g}(80 \%)$. Anal. $\mathrm{C}_{13} \mathrm{H}_{23} \mathrm{BrO}_{2} \mathrm{Si}: \mathrm{C}, \mathrm{H} .{ }^{1} \mathrm{H}$ NMR: $\delta 0.10\left(\mathrm{SiMe}_{2}\right), 0.9$ and $1.10($ thx $) 4.50\left(\mathrm{CH}_{2}\right), 6.30$ $(\mathrm{H}-4), 7.30(\mathrm{H}-2) .{ }^{13} \mathrm{C} \mathrm{NMR:} \delta(50 \mathrm{MHz}): \delta-3.26\left(\mathrm{SiMe}_{2}\right)$, $18.70,20.49,25.41,34.39$ (thx), $57.30\left(\mathrm{CH}_{2} \mathrm{O}\right), 111.53$ (C4), 122.75 (C3), 129.25 (C5), 141.20 (C2). MS: 320/318 $(4 / 4, M), 235$ (100), 233 (100), 207 (42), 205 (42), 161 (12), 159 (12), (84), 137 (75).

2-(Dimethylthexylsilyloxymethyl)furan (4b). Dimethylthexylsilyl chloride $(17.88 \mathrm{~g}, 100 \mathrm{mmol})$ was added dropwise with stirring at $-60^{\circ} \mathrm{C}$ to a solution of freshly distilled 2-furylmethanol $(10.00 \mathrm{~g}, 100 \mathrm{mmol})$, triethylamine $(15.15$ $\mathrm{g}, 150 \mathrm{mmol})$ and DMAP $(0.2 \mathrm{~g})$ in dichloromethane (50 $\mathrm{ml}$ ). The mixture was stirred at $-60^{\circ} \mathrm{C}$ for $2 \mathrm{~h}$, allowed to reach ambient temperature and stirred for $20 \mathrm{~h}$ when TLC showed the reaction to be complete. The mixture was filtered and the solid washed with dichloromethane. The filtrate and washings were shaken with water $(3 \times 100 \mathrm{ml})$ and the organic solution was dried $\left(\mathrm{MgSO}_{4}\right)$, evaporated. The residual oil was distilled to yield a colourless liquid, b.p. $76-78^{\circ} \mathrm{C} / 0.03 \mathrm{mmHg}$, yield $20.00 \mathrm{~g}(83 \%)$. Anal. $\mathrm{C}_{13} \mathrm{H}_{24} \mathrm{O}_{2} \mathrm{Si}: \mathrm{C}, \mathrm{H} .{ }^{1} \mathrm{H}$ NMR: $\delta 0.20\left(\mathrm{SiMe}_{2}\right), 1.0$ (thx), 4.70 $\left(\mathrm{CH}_{2}\right), 6.3(\mathrm{H}-3,4), 7.5(\mathrm{H}-5) .{ }^{13} \mathrm{C}$ NMR: $\delta-3.42\left(\mathrm{SiMe}_{2}\right)$, 18.39, 20.22, 25.21, 34.14 (thx), $57.87\left(\mathrm{CH}_{2} \mathrm{O}\right), 106.85$ (C3), 110.02 (C4), 141.73 (C5), 154.47 (C2). MS(Cl): 241 $(0.7, M+H), 172(7), 159(3), 156(5), 155$ (38), $123(8), 98$ (17), $76(100)$.

3 - Dimethylthexylsilyloxymethyl-5 - (trimethylstannyl)furan (6a). Butyllithium in hexane (1.6 M; $4.40 \mathrm{ml}, 7.04 \mathrm{mmol})$ was added dropwise, by means of a syringe, to a stirred solution of 5-bromo-3-dimethylthexylsilyloxymethyl)furan $(1.77 \mathrm{~g}, 5.55 \mathrm{mmol})$ in dry THF $(25 \mathrm{ml})$ under argon at $-85^{\circ} \mathrm{C}$. The mixture was stirred at $-70^{\circ} \mathrm{C}$ for $60 \mathrm{~min}$, after which the temperature was lowered to $-85^{\circ} \mathrm{C}$ and a solution of trimethylstannyl chloride $(1.40 \mathrm{~g}, 7.04 \mathrm{mmol})$ in dry THF $(5 \mathrm{ml})$ added dropwise with stirring. The mixture was stirred at $-85^{\circ} \mathrm{C}$ for $10 \mathrm{~min}$ before the mixture was allowed to reach ambient temperature and the stirring was continued for $3 \mathrm{~h}$. The mixture was then poured into $10 \%$ aqueous $\mathrm{NH}_{4} \mathrm{Cl}(10 \mathrm{ml})$ and the resultant mixture was stirred vigorously for $5 \mathrm{~min}$. The two phases were separated, the aqueous phase extracted with diethyl ether $(3 \times 20 \mathrm{ml})$, and the combined organic phases were washed with water $(3 \times 20 \mathrm{ml})$, dried $\left(\mathrm{MgSO}_{4}\right)$ and filtered. The filtrate was evaporated and the product was purified by distillation (Kugelrohr); yield $1.80 \mathrm{~g} \quad(80 \%)$, b.p.
$170{ }^{\circ} \mathrm{C} / 0.03 \mathrm{mmHg}$. Anal. $\mathrm{C}_{16} \mathrm{H}_{32} \mathrm{O}_{2} \mathrm{SiSn}: \mathrm{C}, \mathrm{H} .{ }^{1} \mathrm{H}$ NMR: $\delta$ $0.20\left(\mathrm{SiMe}_{2}\right), 0.32\left(\mathrm{SnMe}_{3}\right), 1.0$ and $1.70(\mathrm{thx}), 4.70\left(\mathrm{CH}_{2}\right)$, $6.70(\mathrm{H}-4), 7.70(\mathrm{H}-2)$. MS: $403(11, M), 336(10), 334(5)$, 319 (4), 262 (7), $260(5), 258(6), 256(18), 254$ (11), $186(8)$, 184 (10), 182 (100), 181 (29), 180 (37), 179 (28), 178 (29).

2-Dimethylthexylsilyloxymethyl-5-trimethylstannylfuran (6b). Butyllithium in hexane (1.6 M; $15.62 \mathrm{ml}, 25.0 \mathrm{mmol})$ was added dropwise with stirring by means of a syringe, to a solution of 2-(dimethylthexylsilyloxymethyl)furan $(5.0 \mathrm{~g}$, $20.80 \mathrm{mmol})$ in dry THF $(50 \mathrm{ml})$ under argon at $-60^{\circ} \mathrm{C}$. The mixture was stirred at this temperature for $10 \mathrm{~min}$, after which the temperature was allowed to rise to $20^{\circ} \mathrm{C}$ over $20 \mathrm{~min}$, then lowered to $-60^{\circ} \mathrm{C}$ and a solution of trimethylstannyl chloride $(5.0 \mathrm{~g}, 25.0 \mathrm{mmol})$ in dry THF $(10 \mathrm{ml})$ was added dropwise with stirring. The mixture was stirred at $-60^{\circ} \mathrm{C}$ for $10 \mathrm{~min}$ and the mixture allowed to reach ambient temperature. The stirring was continued for $3 \mathrm{~h}$, after which the mixture was poured into $10 \%$ aqueous $\mathrm{NH}_{4} \mathrm{Cl}(30 \mathrm{ml})$. The two phases were separated, and the aqueous phase was extracted with diethyl ether $(3 \times 50 \mathrm{ml})$. The combined organic phases were washed with water $(4 \times 50 \mathrm{ml})$, dried $\left(\mathrm{MgSO}_{4}\right)$ and filtered, and the filtrate evaporated. The product was purified by distillation; yield $6.20 \mathrm{~g}(74 \%)$, b.p. $92-96^{\circ} \mathrm{C} / 0.005 \mathrm{mmH}$. Anal. $\mathrm{C}_{16} \mathrm{H}_{32} \mathrm{O}_{2}$ SiSn: C, H. ${ }^{1} \mathrm{H}$ NMR: $\delta 0.20\left(\mathrm{SiMe}_{2}\right), 0.40\left(\mathrm{SnMe}_{3}\right)$, 1.0-1.7 (thx), $4.80\left(\mathrm{CH}_{2}\right), 6.40$ and $6.66(\mathrm{H}-3,4) .{ }^{13} \mathrm{C}$ NMR (50 MHz): $\delta-9.11\left(\mathrm{SnMe}_{3}\right),-3.13\left(\mathrm{SiMe}_{2}\right), 18.71,20.52$, $25.46,34.41$ (thx), $58.45\left(\mathrm{CH}_{2} \mathrm{O}\right), 107.46(\mathrm{C} 4), 122.03$ (C-3), 159.65 (C5), 160.65 (C2). MS: 408/407/406/405/404/ 403/402/401 (3/6/11/15/13/15/12/15, M), 387 (15), 383 (16), 336 (46), 335 (42), 334 (30), 332 (21), 329 (20), 326 (33), 325 (72), 324 (42), 323 (58), 322 (28), 321 (45), 255 (31), 245 (100), 243 (74), 242 (30), 241 (52), 182 (81), 180 (55), $178(34)$.

2-Methylthio-5-(4-dimethylthexylsilyloxymethyl-2-furoyl)pyrimidine (8a). A mixture of bis(triphenylphospine)palladium(II) dichloride $(0.80 \mathrm{~g}, 1.1 \mathrm{mmol}), 2$-methylthiopyrimidine-5-carbonyl chloride $(2.82 \mathrm{~g}, 15.0 \mathrm{mmol})$ and 3-(dimethylthexylsilyloxymethyl-5-trimethylstannyl)furan $(5.83$ $\mathrm{g}, 14.4 \mathrm{mmol})$, in dry THF $(120 \mathrm{ml})$ was heated under reflux for $30 \mathrm{~min}$ when TLC monitoring showed that the reaction had gone to completion. The reaction mixture was concentrated to a quarter of its volume and diethyl ether added to the cold mixture. The washed (aqueous $\mathrm{NaCl}$; $3 \times 20 \mathrm{ml})$ and dried $\left(\mathrm{MgSO}_{4}\right)$ solution was evaporated and the product purified by flash chromatography on silica gel using light petroleum-EtOAc (4:1). The product was an oil; yield $4.34 \mathrm{~g}(73 \%)$. Anal. $\mathrm{C}_{19} \mathrm{H}_{28} \mathrm{~N}_{2} \mathrm{O}_{3} \mathrm{SSi}: \mathrm{C}, \mathrm{H}$. ${ }^{1} \mathrm{H}$ NMR: $\delta 0.10\left(\mathrm{SiMe}_{2}\right), 1.8$ and 1.40 (thx), 2.60 (SMe), 4.55 $\left(\mathrm{CH}_{2}\right), 7.20\left(\mathrm{H}-5^{\prime}\right), 7.55\left(\mathrm{H}-2^{\prime}\right), 9.05$ (H-4,6). ${ }^{13} \mathrm{C}$ NMR: $\delta$ -3.62 $\left(\mathrm{SiMe}_{2}\right), 14.07$ (SMe), 18.30, 20.10, 25.03, 33.98 (thx), $56.55\left(\mathrm{CH}_{2} \mathrm{O}\right), 113.32\left(\mathrm{C}^{\prime}\right), 119.25\left(\mathrm{C}^{\prime}\right), 129.19$ (C5), $143.88\left(\mathrm{C5}^{\prime}\right), 157.59(\mathrm{C} 4,6), 152.30\left(\mathrm{C}^{\prime}\right), 176.64$ (C2), $177.58(\mathrm{C}=\mathrm{O}$, ketone). $\mathrm{MS}(\mathrm{Cl}): 393(100, M+\mathrm{H})$, 379 (3), 324 (4), 313 (3), 309 (6), 307 (7), 279 (2), 251 (10). 
2-Methylthio-5-(5-dimethylthexylsilyloxymethyl-2-furoyl)pyrimidine $(\mathbf{8 b})$. Compound $\mathbf{8 b}$ was prepared as above from 2-methylthiopyrimidine-5-carbonyl chloride (15 mmol), 2-(dimethylthexylsilyloxymethyl-5-trimethylstannyl)furan $(14.4 \mathrm{mmol})$ in the presence of bis(triphenylphosphine)palladium(II) dichloride $(1.1 \mathrm{mmol})$ in THF $(120 \mathrm{ml})$. The mixture was heated under reflux for $30 \mathrm{~min}$ and the product was purified as described above. The product was a pale yellow oil; yield $77 \%$. Anal. $\mathrm{C}_{19} \mathrm{H}_{28} \mathrm{~N}_{2} \mathrm{O}_{3} \mathrm{SSi}$ : C, H. ${ }^{1} \mathrm{H}$ NMR: $\delta 0.10\left(\mathrm{SiMe}_{2}\right), 1.8$ and 1.40 (thx), 2.60 (SMe), $4.55\left(\mathrm{CH}_{2}\right), 7.20\left(\mathrm{H}^{\prime} 5^{\prime}\right), 7.55\left(\mathrm{H}-3^{\prime}\right), 9.05(\mathrm{H}-4,6)$. ${ }^{13} \mathrm{C}$ NMR: $\delta-3.62\left(\mathrm{SiMe}_{2}\right), 14.07$ (SMe), 18.30, 20.10, 25.03, 33.98 (thx), $56.55\left(\mathrm{CH}_{2} \mathrm{O}\right), 113.32\left(\mathrm{C}^{\prime}\right), 119.25$ (C3'), 129.19 (C5), 148.88 (C5'), 157.59 (C4,6), 152.30 (C2'), 176.64 (C2), 177.58 (C=O, ketone). $\mathrm{MS}(\mathrm{Cl}): 393$ (100, M+H), 379 (3), 324 (4), 313 (3), 309 (6), 307 (7), $279(2), 251(10)$.

2-Methylsulfonyl-5-(4-dimethylthexylsilyloxymethyl-2-furoyl)pyrimidine (9a). A solution of 2-methylthio-5-(4-dimethylthexylsilyloxymethyl-2-furoyl)pyrimidine $(1.20 \mathrm{~g}$, $3.06 \mathrm{mmol})$ and MCPBA (1.55 g, $9.0 \mathrm{mmol})$ in dichloromethane $(80 \mathrm{ml})$ was stirred at ambient temperature for $3 \mathrm{~h}$ when TLC monitoring showed the reaction to be complete. The mixture was then shaken with saturated aqueous sodium hydrogen sulfite $(3 \times 40 \mathrm{ml})$, and then with saturated aqueous sodium hydrogen carbonate $(3 \times 40 \mathrm{ml})$ and the solution dried $\left(\mathrm{MgSO}_{4}\right)$ and evaporated. The residual product was purified by flash chromatography on silica gel using light petroleum-EtOAc (1:1); yield $1.20 \mathrm{~g} \mathrm{(81 \% )}$ of an oily material. ' $\mathrm{H}$ NMR: $\delta 0.10\left(\mathrm{SiMe}_{2}\right)$ and 1.5 (thx), $3.30\left(\mathrm{SO}_{2} \mathrm{Me}\right), 4.70\left(\mathrm{CH}_{2}\right), 7.40\left(\mathrm{H}-3^{\prime}\right), 7.70\left(\mathrm{H}-5^{\prime}\right), 9.50$ (H-4,6). ${ }^{13} \mathrm{C}$ NMR: $\delta-3.65\left(\mathrm{SiMe}_{2}\right), 18.28,20.09,25.04$, 33.94 (thx), $39.02\left(\mathrm{SO}_{2} \mathrm{Me}\right), 56.42\left(\mathrm{CH}_{2} \mathrm{O}\right), 113.88\left(\mathrm{C}^{\prime}\right)$, $120.71\left(\mathrm{C}^{\prime}\right), 129.96$ (C5), $145.09\left(\mathrm{C}^{\prime}\right), 151.64\left(\mathrm{C}^{\prime}\right)$, $159.01(\mathrm{C} 4,6), 167.23$ (C2), 179.98 (C=O, ketone). MS(Cl): $442(100, M+\mathrm{H}), 427$ (6), $426(14), 425$ (46), 395 (8), 393 (29), 363 (5), 362 (11), 356 (7).

2-Methylsulfonyl-5-(5-dimethylthexylsilyloxymethyl-2-furoyl)pyrimidine (9b). Compound 9b was prepared from 2-methylthio-5-(5-dimethylthexylsilyloxymethyl-2-furoyl)pyrimidine by oxidation with MCPBA as described above; yield $92 \%$, non-crystalline solid. Anal. $\mathrm{C}_{19} \mathrm{H}_{28} \mathrm{~N}_{2} \mathrm{O}_{5} \mathrm{SSi}$ : C, H. ${ }^{1} \mathrm{H}$ NMR: $\delta 0.13\left(\mathrm{SiMe}_{2}\right), 0.9$ and 1.50 (thx), 3.40 $\left(\mathrm{SO}_{2} \mathrm{Me}\right), 4.80\left(\mathrm{CH}_{2}\right), 6.60\left(\mathrm{H}^{4}{ }^{\prime}, \mathrm{d}, J 4.0 \mathrm{~Hz}\right), 7.50\left(\mathrm{H}^{-3} 3^{\prime}\right.$, d), $9.40(\mathrm{H}-4,6) .{ }^{13} \mathrm{C} \mathrm{NMR:} \delta-3.32\left(\mathrm{SiMe}_{2}\right), 18.67,20.41$, 25.45, 34.33 (thx), $39.48\left(\mathrm{SO}_{2} \mathrm{Me}\right), 58.72\left(\mathrm{CH}_{2} \mathrm{O}\right), 110.82$ (C4'), 123.78 (C3'), 132.79 (C5), 151.27 (C5'), 159.65 (C4,6), $163.03\left(\mathrm{C} 2^{\prime}\right), 167.93(\mathrm{C} 2), 176.52$ (C=O, ketone). MS (Cl): $425(100, M+H), 409$ (4), 356 (7), $341(10), 340$ (15), 339 (72), 265 (6), 233 (3).

5-(4-Dimethylthexylsilyloxymethyl-2-furoyl)-2(1H)-pyrimidinone (10a). $2 \mathrm{M} \mathrm{NaOH}(12 \mathrm{ml})$ was added to a mixture of 2-methylsulfonyl-5-(4-dimethylthexylsilyloxymethyl-2-fur- oyl)pyrimidine $(3.26 \mathrm{~g}, 7.7 \mathrm{mmol})$ in dioxane-water (1:1; $40 \mathrm{ml}$ ), and the mixture stirred at ambient temperature for $15 \mathrm{~min}$ when all the solid had dissolved. The $\mathrm{pH}$ of the solution was adjusted to ca. 6 by gradual addition of acetic acid whereupon the product precipitated; yield $1.95 \mathrm{~g}$ (70\%), m.p. $160{ }^{\circ} \mathrm{C}$. Anal. $\mathrm{C}_{18} \mathrm{H}_{26} \mathrm{~N}_{2} \mathrm{O}_{4} \mathrm{Si}: \mathrm{C}, \mathrm{H} .{ }^{1} \mathrm{H}$ NMR: $\delta 0.10\left(\mathrm{SiMe}_{2}\right), 0.8$ and 1.7 (thx), $4.60\left(\mathrm{CH}_{2}\right), 7.30\left(\mathrm{H}-5^{\prime}\right)$, $7.60\left(\mathrm{H}-3^{\prime}\right), 9.20(\mathrm{H}-4,6) .{ }^{13} \mathrm{C}$ NMR: $\delta-3.40\left(\mathrm{SiMe}_{2}\right)$, 18.52, 20.31, 25.23, 34.16, (thx), $56.77\left(\mathrm{CH}_{2} \mathrm{O}\right), 115.60$ (C4'), 119.22 (C3'), 129.51 (C5), 143.74 (C5'), 152.39 (C2'), 158.09 (C4,6), 159.37 (C2), 174.75 (C=O, ketone). MS (Cl): $363(100, M+H), 361$ (3), 295 (2), 294 (4), 279 (7), 278 (4), 277 (12), 222 (2), 221 (2).

5-(5-Dimethylthexylsilyloxymethyl-2-furoyl)-2(1H)-pyrimidinone (10b). Compound 10b was prepared from 2methylsulfonyl-5-(5-dimethylthexylsilyloxymethyl-2-furoyl)pyrimidine by hydrolysis with $2 \mathrm{M} \mathrm{NaOH}$ as described above; yield $82 \%$, m.p. $178-180^{\circ} \mathrm{C}$. Anal. $\mathrm{C}_{18} \mathrm{H}_{26} \mathrm{~N}_{2} \mathrm{O}_{4} \mathrm{Si}$ : C, H. ${ }^{1} \mathrm{H}$ NMR: $\delta 0.13\left(\mathrm{SiMe}_{2}\right), 0.9$ and 1.6 (thx), 4.80 $\left(\mathrm{CH}_{2}\right), 6.50\left(\mathrm{H}-4^{\prime}, \mathrm{d}, J 4.0 \mathrm{~Hz}\right), 7.40\left(\mathrm{H}-3^{\prime}, \mathrm{d}\right), 9.20$ $(\mathrm{H}-4,6) .{ }^{13} \mathrm{C}$ NMR $(50 \mathrm{MHz}): \delta-3.29\left(\mathrm{SiMe}_{2}\right), 18.69$, $20.45,25.47,34.36$ (thx), $58.86\left(\mathrm{CH}_{2} \mathrm{O}\right), 110.19\left(\mathrm{C}^{\prime}\right)$, 116.34 (C5), $121.84\left(\mathrm{C3}^{\prime}\right), 151.73\left(\mathrm{C}^{\prime}\right), 158.92\left(\mathrm{C}^{\prime}\right), 160$ (C2), $161.36(\mathrm{C} 4,6), 171.11(\mathrm{C}=\mathrm{O}$, ketone). $\mathrm{MS}(\mathrm{Cl}): 363$ (100, M+H), 294 (5), 279 (4), 278 (9), 277 (44), 249 (5).

5-(4-Hydroxymethyl-2-furoyl)-2(1H)-pyrimidinone (11a). Tetrabutylammonium fluoride $(0.5 \mathrm{M}$ solution in THF; $13.6 \mathrm{ml}, 6.79 \mathrm{mmol})$ was added dropwise by means of a syringe to a solution of 5-(4-dimethylthexylsilyloxymethyl-2-furoyl)-2(1H)-pyrimidinone $(0.82 \mathrm{~g}, 2.20 \mathrm{mmol})$ in THF $(10 \mathrm{ml})$ at $0^{\circ} \mathrm{C}$ under argon. The mixture was stirred at ambient temperature for $2 \mathrm{~h}$, cooled to ca. $5^{\circ} \mathrm{C}$ and acetic acid added slowly until precipitation of the product was complete; yield $0.38 \mathrm{~g}(78 \%)$, m.p. $273^{\circ} \mathrm{C}$. Anal. $\mathrm{C}_{10} \mathrm{H}_{8} \mathrm{~N}_{2} \mathrm{O}_{4}: \mathrm{C}, \mathrm{H}$. ${ }^{1} \mathrm{H}$ NMR (DMSO- $d_{6}$ ): $\delta 5.12$ $\left(\mathrm{CH}_{2} \mathrm{O}\right), 8.14\left(\mathrm{H}^{-} 3^{\prime}\right), 8.66\left(\mathrm{H}-5^{\prime}\right), 9.57$ (H-4,6). ${ }^{13} \mathrm{C}$ NMR (DMSO-d $\left.d_{6}\right): \delta 54.42\left(\mathrm{CH}_{2} \mathrm{O}\right), 113.71\left(\mathrm{C}^{\prime}\right), 119.56\left(\mathrm{C}^{\prime}\right)$, 129.35 (C5), $144.50\left(\mathrm{C}^{\prime}\right), 151.26\left(\mathrm{C} 2^{\prime}\right), 155.73(\mathrm{C} 2)$, $158.61(\mathrm{C} 4,6), 175.35(\mathrm{C}=\mathrm{O}$, ketone $)$. MS: $220(100, M)$, 219 (11), 218 (52), 202 (49), 192 (11), 191 (31), 190 (11), 174 (30), 173 (15), 163 (18), 162 (20).

5-(5-Hydroxymethyl-2-furoyl)-2(1H)-pyrimidinone (11b). Compound 11b was prepared from 5-(5-dimethylthexylsilyloxymethyl-2-furoyl)-2(1H)-pyrimidinone by cleavage of the dimethylthexylsilyl group with tetrabutylammonium fluoride as described above; yield $82 \%$, m.p. $278-280^{\circ} \mathrm{C}$ $\left(\mathrm{H}_{2} \mathrm{O}\right)$. Anal. $\mathrm{C}_{10} \mathrm{H}_{8} \mathrm{~N}_{2} \mathrm{O}_{4}$ : C, H. ${ }^{1} \mathrm{H}$ NMR (DMSO- $d_{6}$ ): $\delta$ $4.65\left(\mathrm{CH}_{2}\right), 5.5(\mathrm{OH}), 6.71\left(\mathrm{H}-4^{\prime}, \mathrm{d}, J 3.4 \mathrm{~Hz}\right), 7.55\left(\mathrm{H}-3^{\prime}\right.$, d), $9.00(\mathrm{H}-4,6) .{ }^{13} \mathrm{C}$ NMR (DMSO- $\left.d_{6}\right): \delta 56.02\left(\mathrm{CH}_{2} \mathrm{O}\right)$, 109.89 (C4'), 113.84 (C5), 121.09 (C3'), 150.32 (C5'), 155.41 (C2'), $160.00(\mathrm{C} 2), 161.11(\mathrm{C} 4,6), 175.15$ (C=O, ketone). MS: $220(100, M), 219(1), 218(2), 203(6), 191$ (10), 189 (73), 163 (10), 161 (17). 
1-Benzyl-5-(4-hydroxymethyl-2-furoyl)-2(1H)-pyrimidinone (12a). A mixture of triethylamine $(0.07 \mathrm{ml}, 0.04 \mathrm{mmol})$ and 5-(4-hydroxymethyl-2-furoyl)-2(1H)-pyrimidinone $(61 \mathrm{mg}$, $0.28 \mathrm{mmol})$ in dichloromethane $(3 \mathrm{ml})$ was stirred for 15 min after which time all the solid had dissolved. Benzyl bromide ( $84 \mathrm{mg}, 0.49 \mathrm{mmol}$ ) was added and the mixture was stirred at ambient temperature for $2 \mathrm{~h}$ when TLC showed the reaction to be complete. The mixture was evaporated and the residue purified by flash chromatography on silica gel using $\mathrm{CHCl}_{3}$-diethyl ether-ethanol $(5: 4: 1)$ to give a white product; yield $71 \mathrm{mg}(82 \%)$, m.p. $167^{\circ} \mathrm{C}$. Anal. $\mathrm{C}_{17} \mathrm{H}_{14} \mathrm{~N}_{2} \mathrm{O}_{4}:$ C, H. ${ }^{1} \mathrm{H}$ NMR (DMSO- $\left.d_{6}\right): \delta 4.54\left(\mathrm{CH}_{2}, \mathrm{~d}, J\right.$ $5.7 \mathrm{~Hz}), 5.57(\mathrm{OH}, \mathrm{t}, J 5.7 \mathrm{~Hz}), 5.20\left(\mathrm{NCH}_{2}\right), 6.46\left(\mathrm{H}-4^{\prime}\right.$, d, J 3.3 Hz), $7.46\left(\mathrm{H}-3 '^{\prime}, \mathrm{d}\right), 7.3-7.4(\mathrm{Ph}), 9.09$ (H-6, d, J $3.3 \mathrm{~Hz}$ ), $9.13(\mathrm{H}-4, \mathrm{~d}) .{ }^{13} \mathrm{C}$ NMR (DMSO- $\left.d_{6}\right): \delta 53.93$ $\left(\mathrm{CH}_{2} \mathrm{OH}\right), 54.29\left(\mathrm{NCH}_{2}\right), 114.37\left(\mathrm{C4}^{\prime}\right), 119.98\left(\mathrm{C3}^{\prime}\right)$, 129.30 (C5), 128.03, 128.54, 128.64, $135.43(\mathrm{Ph}), 144.71$ (C5'), 150.80 (C6), 153.43 (C2'), 154.38 (C2), 165.30 (C4), 175.06 (C=O, ketone). MS: $310(66, M), 409(7), 308(20)$, 221 (9), 219 (23), 201 (20), 125 (14), 92 (17), 91 (100).

1-Benzyl-5-(5-hydroxymethyl-2-furoyl)-2(1H)-pyrimidinone (12b). Compound 12b was prepared from triethylamine and 5-(5-hydroxymethyl-2-furoyl)-2(1H)-pyrimidinone by alkylation with benzyl bromide as described above; yield $82 \%$, m.p. $167^{\circ} \mathrm{C}$. Anal. $\mathrm{C}_{17} \mathrm{H}_{14} \mathrm{~N}_{2} \mathrm{O}_{4}$ : C, H. ${ }^{1} \mathrm{H}$ NMR (DMSO-d $d_{6}$ ): $\delta 4.54\left(\mathrm{CH}_{2}, \mathrm{~d}, J 5.7 \mathrm{~Hz}\right), 5.57(\mathrm{OH}, \mathrm{t}, J 5.7$

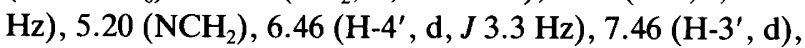

7.3-7.4 (Ph), 9.09 (H-6, d, J 3.3 Hz), $9.13(\mathrm{H}-4, \mathrm{~d}) .{ }^{13} \mathrm{C}$ NMR (DMSO- $\left.d_{6}\right): \delta 53.93\left(\mathrm{CH}_{2} \mathrm{OH}\right), 55.86\left(\mathrm{NCH}_{2}\right)$, 109.52 (C4'), 114.48 (C5), 121.35 (C3'), 127.94, 128.14, 128.56, $135.47(\mathrm{Ph}), 149.84(\mathrm{C} 6), 153.31\left(\mathrm{C5}^{\prime}\right), 154.42$ (C2'), 161.24 (C2), 165.29 (C4), 174.85 (C=O, ketone). MS: 310 (4, M), 308 (34), 279 (7), 251 (4), 217 (5), 123 (7), 92 (11), 91 (100).

\section{References}

1. Arukwe, J. and Undheim, K. Acta Chem. Scand., Ser. B 40 (1986) 588.

2. Arukwe, J. and Undheim, K. Acta Chem. Scand., Ser. B 40 (1986) 764.

3. Arukwe, J., Benneche, T. and Undheim, K. J. Chem. Soc., Perkin Trans. 1 (1989) 255.

4. Undheim, K. In: van der Goot, H., Domány, G., Pallos, L. and Timmerman, H., Eds. Trends in Medicinal Chemistry '88, Elsevier, Amsterdam 1989, p. 781.

5. Benneche, T., Keilen, G., Hagelin, G., Oftebro, R. and Undheim, K. Acta Chem. Scand. 45 (1991) 177.

6. Oftebro, R. and Undheim, K. Unpublished data.

7. Wetter, H. and Oertle, K. Tetrahedron Lett. 26 (1985) 5515.

8. Bures, E. J. and Keay, B. A. Tetrahedron Lett. 28 (1987) 596.

9. Tanis, S. P. and head, D. B. Tetrahedron Lett. 25 (1984) 4451.

10. (a) Fournari, P., Meunier, J. M. and Sornay, R. Bull. Soc. Chim. Fr. (1971) 990; (b) do Amaral, L. and Ferras, J. P. J. Org. Chem. 41 (1976) 2350.

11. Amstutz, E. D., and Sherman, E. J. Am. Chem. Soc. 72 (1950) 2195.

Received November 28, 1990. 